International Journal of Applied Mathematics

Volume 32 No. $6 \quad 2019,1069-1082$

ISSN: 1311-1728 (printed version); ISSN: 1314-8060 (on-line version)

doi: http://dx.doi.org/10.12732/ijam.v32i6.12

\title{
ECCENTRICITY BASED ZAGREB INDICES OF BISMUTH TRI-IODIDE
}

\author{
Hai-Xia $\mathrm{Li}^{1}$, Abdul Qudair Baig ${ }^{2}$, \\ Muhammad Razwan Azhar ${ }^{2}$ \\ ${ }^{1}$ Department of Mathematics and Physics \\ Anhui Xinhua University, Hefei, CHINA \\ ${ }^{2}$ Department of Mathematics \\ COMSATS Institute of Information Technology \\ Attock, PAKISTAN
}

\begin{abstract}
Graph theory has much advancement in the field of mathematical chemistry. Now a days, chemical graph theory has become very popular among researchers because of its wide applications in mathematical chemistry. The molecular topological descriptors are the numerical invariants of a molecular graph and are very useful for predicting their bioactivity. A great variety of such indices are studied and used in theoretical chemistry, pharmaceutical researchers, in drugs and in different other fields. In this article, we study the chemical graph of Bismuth-tri-iodide and compute the eccentricity based Zagreb indices for Bismuth-tri-iodide. Furthermore, we give analytically closed formulas of these indices which are helpful in studying the underlying topologies.
\end{abstract}

AMS Subject Classification: 05C12, 05C90

Key Words: eccentricity based Zagreb index, Molecular graph, Bismuth-triiodide

\section{Introduction}

There are a lot of chemical compounds, either organic or inorganic, which pos-

Received: June 17, 2019

(c) 2019 Academic Publications

${ }^{\S}$ Correspondence author 
sess a level of commercial, industrial, pharmaceutical chemistry and laboratory importance. A relationship exists between chemical compounds and their molecular structures. Graph theory is a very powerful area of mathematics that has wide range of applications in many areas of science such as chemistry, biology, computer science, electrical, electronics and other fields. Chemical graph theory is a branch of mathematical chemistry in which we apply tools of graph theory to model the chemical phenomenon mathematically. This theory contributes a prominent role in the field of chemical sciences. Some references are given, which hopefully demonstrate the importance of this field $[19,20,16,17,18,12,1,14,2]$.

Consider $G=(V, E)$ be a graph, where $V$ is a non-empty set of vertices and $E$ is a set of edges. The chemical graph theory applies graph theory to mathematical modeling of molecular phenomena, which is helpful for the study of molecular structure. This theory contributes a prominent role in the field of chemical sciences. Chemical compounds have a variety of applications in chemical graph theory, drug design, etc. The manipulation and examination of chemical structural information is made conceivable by using molecular descriptors. A great variety of topological indices are studied and used in theoretical chemistry, pharmaceutical researchers. In chemical graph theory, there are many topological indices for a connected graph, which are helpful in study of chemical molecules. Development of chemical science had an important effect by this theory.

If $p, q \in V(G)$, then the distance $d(p, q)$ between $p$ and $q$ is defined as the length of any shortest path in $G$ connecting $p$ and $q$. Eccentricity is the distance of vertex $u$ from the farthest vertex in $G$. In mathematical form,

$$
\varepsilon(u)=\max \{d(u, v) \mid \forall u \in V(G)\} .
$$

Recently in 2010, D. Vukiçević et al., and in 2012, Ghorbani et al. proposed some new modified versions of Zagreb indices of a molecular graph $G,[11,4]$.

These indices are eccentricity based indices, which are defined as

$$
\begin{gathered}
M_{1}^{*}(G)=\sum_{u v \in E(G)}[\varepsilon(u)+\varepsilon(v)], \\
M_{1}^{* *}(G)=\sum_{v \in V(G)}[\varepsilon(v)]^{2}, \\
M_{2}^{*}(G)=\sum_{u v \in E(G)}[\varepsilon(u) \cdot \varepsilon(v)] .
\end{gathered}
$$


Some applications of eccentricity based Zagreb indices are given in $[7,8,9,3]$.

Bismuth tri-iodide $\left(\mathrm{BiI}_{3}\right)$ is an inorganic compound. It is the product of the reaction of bismuth and iodine, which once was of interest in qualitative inorganic analysis. Layered $\mathrm{BiI}_{3}$ crystal is considered to be a three-layered stacking structure, where bismuth atom planes are sandwiched between iodide atom planes, which form the sequence $I-B i-I$ planes. The periodic stacking of three layers forms rhombohedral $\mathrm{BiI}_{3}$ crystal with $R-3$ symmetry [10, 15]. The successive stacking of one $I-B i-I$ layer forms hexagonal structure with symmetry [6, 13]. A single crystal of $\mathrm{BiI}_{3}$ has been synthesized by Nason and Keller [5]. Fig. 1 shows one unit of bismuth tri-iodide.

Graph of a single unit of bismuth tri-iodide contains six 4-cycles of which two are on the top, two are in the middle and two at the bottom. The unit cells of bismuth tri-iodide can be arranged either linearly or in a sheet form. A linear arrangement with $m$ unit cells is called an $m$-bismuth chain; $m n$ unit cells arranged into $m$ rows and $\mathrm{n}$ columns is called an $m \times n$ bismuth sheet. Fig. 2 shows $2 \times 6$ bismuth sheet.

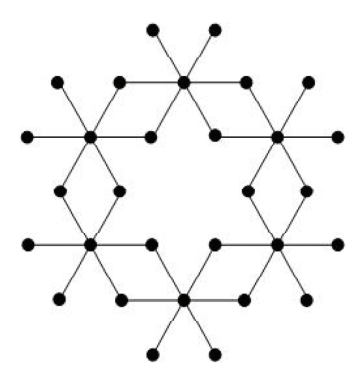

Figure 1: One unit of Bismuth tri-iodide. 


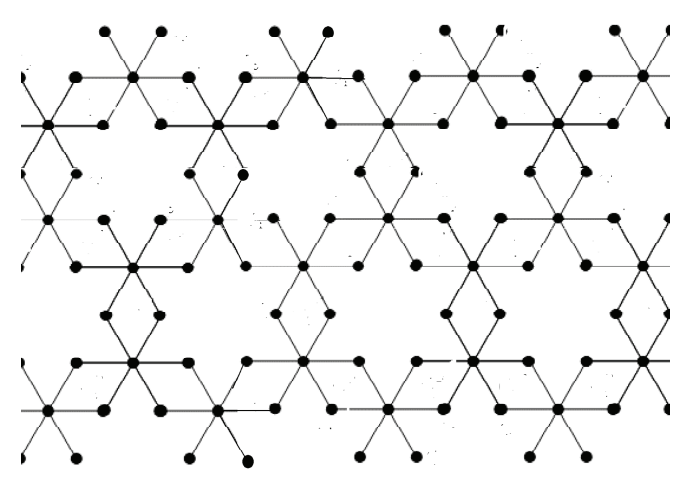

Figure 2: Bismuth sheet of order $2 \times 6$.

\section{Main results and discussion}

In this section, we discussed the eccentricity based Zagreb indices $M_{1}^{*}(G)$, $M_{1}^{* *}(G)$ and $M_{1}^{*}(G)$ of Bismuth tri-iodide. Here we consider the Bismuth triiodide graph $\mathrm{BiI}_{3}=G$. The cardinality of vertices and edges in $\mathrm{BiI}_{3}$ are $2(4 m n+5 n+5 m+1)$ and $12(m+m n+n)$, respectively.

\subsection{Eccentricity based first Zagreb index}

In this section we find the first Zagreb eccentricity index of Bismuth tri-iodide $M_{1}^{*}(G(m, n))$.

Theorem 1. Let $G(m, n)$, for all $m, n \in N$, where $m$ and $n$ have opposite pairity, be the bismuth tri-iodide, then the first Zagreb eccentricity index $M_{1}^{*}$ of $G(m, n)$ is

$$
\begin{aligned}
& M_{1}^{*}(G(m, n))=4 \sum_{m \geq 1}\left\{(2 m-1)(4 m+4 n+1)+(m+1) \sum_{k=1}^{\frac{n-m+3}{2}}(8 k+4 m+\right. \\
& \left.4 n-5)+(m+1) \sum_{k=1}^{\frac{n-m+1}{2}}(40 k+20 m+20 n-3)\right\}+4 \sum_{m \geq 2} \sum_{p=0}^{m-2}
\end{aligned}
$$




\begin{tabular}{|c|c|c|}
\hline$(\varepsilon(u), \varepsilon(v))$ & frequency & Range of $k$ and $p$ \\
\hline$(k, k+1)$ & $4(2 m-1)$ & $k=2 m+2 n$ \\
\hline$(s-3, s-2)$ & $4(m+1)$ & $1 \leq k \leq \frac{n-m+3}{2}$ \\
\hline$(s-2, s-1)$ & $4(m+1)$ & $1 \leq k \leq \frac{n-m+1}{2}$ \\
\hline$(s-1, s)$ & $8(m+1)$ & $1 \leq k \leq \frac{n-m+1}{2}$ \\
\hline$(s, s+1)$ & $8(m+1)$ & $1 \leq k \leq \frac{n-m+1}{2}$ \\
\hline$(k, k+1)$ & $4(m-p)$ & $k=4 n+4 p+4,0 \leq p \leq m-2$ \\
\hline$(k, k+1)$ & $8(m-p)$ & $k=4 n+4 p+5,0 \leq p \leq m-2$ \\
\hline$(k, k+1)$ & $8(m-p-1)$ & $k=4 n+4 p+6,0 \leq p \leq m-2$ \\
\hline$(k, k+1)$ & $4(m-p-1)$ & $k=4 n+4 p+7,0 \leq p \leq m-2$ \\
\hline
\end{tabular}

Table 1: Edge partition of Bismuth tri-Iodide for $((m, n)$-levels $)$ where $m$ and $n$ have opposite pairity and $m \geq 2, n \geq m+1$, based on eccentricity of end vertices of each edge with existence of their frequencies (Let $s=4 k+2 m+2 n$ ).

\begin{tabular}{|c|c|c|}
\hline$(\varepsilon(u), \varepsilon(v))$ & frequency & Range of $k$ and $p$ \\
\hline$(k, k+1)$ & $4(m-1)$ & $k=2 m+2 n$ \\
\hline$(k, k+1)$ & $4(2 m+1)$ & $k=2 m+2 n+1$ \\
\hline$(s-2, s-1)$ & $8(m+1)$ & $1 \leq k \leq \frac{n-m+2}{2}$ \\
\hline$(s-1, s)$ & $4(m+1)$ & $1 \leq k \leq \frac{n-m+2}{2}$ \\
\hline$(s, s+1)$ & $4(m+1)$ & $1 \leq k \leq \frac{n-m}{2}$ \\
\hline$(s+1, s+2)$ & $8(m+1)$ & $1 \leq k \leq \frac{n-m}{2}$ \\
\hline$(k, k+1)$ & $4(m-p)$ & $k=4 n+4 p+4,0 \leq p \leq m-2$ \\
\hline$(k, k+1)$ & $8(m-p)$ & $k=4 n+4 p+5,0 \leq p \leq m-2$ \\
\hline$(k, k+1)$ & $8(m-p-1)$ & $k=4 n+4 p+6,0 \leq p \leq m-2$ \\
\hline$(k, k+1)$ & $4(m-p-1)$ & $k=4 n+4 p+7,0 \leq p \leq m-2$ \\
\hline
\end{tabular}

Table 2: Edge partition of Bismuth tri-Iodide for $((m, n)$-levels $)$ where $m$ and $n$ have same pairity and $m \geq 2, n \geq m+2$, based on eccentricity of end vertices of each edge with existence of their frequencies.

$\{(m-p)(48 n+48 p+72)-(24 n+24 p+41)\}$.

Proof. Let $G(m, n)$, where $m$ and $n$ have opposite pairity, be the bismuth tri-iodide contains $2(4 m n+5 n+5 m+1)$ vertices and $12(m+m n+n)$ edges. 


\begin{tabular}{|c|c|c|}
\hline$\varepsilon(u)$ & frequency & Range of $k$ and $p$ \\
\hline$k$ & $2(2 m-1)$ & $k=2 m+2 n$ \\
\hline$k$ & $2 m$ & $k=2 m+2 n+1$ \\
\hline$s-2$ & $4(m+1)$ & $1 \leq k \leq \frac{n-m+3}{2}$ \\
\hline $2 k+1$ & $2(m+1)$ & $m+n+1 \leq k \leq 2 n+1$ \\
\hline$s$ & $4(2 m+3)$ & $1 \leq k \leq \frac{n-m+1}{2}$ \\
\hline$k$ & $2(m-p)$ & $k=4 n+4 p+5,0 \leq p \leq m-2$ \\
\hline$k$ & $8(m-p)$ & $k=4 n+4 p+6,0 \leq p \leq m-2$ \\
\hline$k$ & $2(m-p-1)$ & $k=4 n+4 p+7,0 \leq p \leq m-2$ \\
\hline$k$ & $4(m-p-1)$ & $k=4 n+4 p+8,0 \leq p \leq m-2$ \\
\hline
\end{tabular}

Table 3: Vertex partition of Bismuth tri-Iodide for $((m, n)$-levels $)$ where $m$ and $n$ have opposite pairity and $m \geq 2, n \geq m+1$, based on eccentricity of each vertex with existence of their frequencies.

\begin{tabular}{|c|c|c|}
\hline$\varepsilon(u)$ & frequency & Range of $k$ and $p$ \\
\hline$k$ & $2(m-1)$ & $k=2 m+2 n$ \\
\hline$k$ & $2 m$ & $k=2 m+2 n+1$ \\
\hline$k$ & $8(m+1)$ & $k=2 m+2 n+2$ \\
\hline $2 k+1$ & $2(m+1)$ & $m+n+1 \leq k \leq 2 n+1$ \\
\hline$s$ & $4(m+1)$ & $1 \leq k \leq \frac{n-m+2}{2}$ \\
\hline$s+2$ & $4(2 m+3)$ & $1 \leq k \leq \frac{n-m}{2}$ \\
\hline$k$ & $2(m-p)$ & $k=4 n+4 p+5,0 \leq p \leq m-2$ \\
\hline$k$ & $8(m-p)$ & $k=4 n+4 p+6,0 \leq p \leq m-2$ \\
\hline$k$ & $2(m-p-1)$ & $k=4 n+4 p+7,0 \leq p \leq m-2$ \\
\hline$k$ & $4(m-p-1)$ & $k=4 n+4 p+8,0 \leq p \leq m-2$ \\
\hline
\end{tabular}

Table 4: Vertex partition of Bismuth tri-Iodide for $((m, n)$-levels $)$ where $m$ and $n$ have same pairity and $m \geq 2, n \geq m+2$, based on eccentricity of each vertex with existence of their frequencies.

The formula of first Zagreb eccentricity index is:

$$
M_{1}^{*}(G)=\sum_{u v \in E(G)}[\varepsilon(u)+\varepsilon(v)] .
$$

Using the edge partitioned from Table 1, we have the following computations: $M_{1}^{*}\left(G(m, n)=\sum_{m \geq 1}\left\{4(2 m-1) \sum_{k=2 m+2 n}(k+k+1)+4(m+1) \sum_{k=1}^{\frac{n-m+3}{2}}(4 k+\right.\right.$ $2 m+2 n-3+4 k+2 m+2 n-2)+4(m+1) \sum_{k=1}^{\frac{n-m+1}{2}}\{(4 k$ 
$+2 m+2 n-2+4 k+2 m+2 n-1)+2(4 k+2 m+2 n-1+4 k+2 m+2 n)+$ $2(4 k+2 m+2 n+1+4 k+2 m+2 n)\}\}+4 \sum_{m \geq 2} \sum_{p=0}^{m-2}\left\{(m-p)\left\{\sum_{k=4 n+4 p+4}(k+\right.\right.$ $\left.k+1)+2 \sum_{k=4 n+4 p+5}(k+k+1)\right\}+(m-p-1)\left\{2 \sum_{k=4 n+4 p+6}(k+k+1)+\right.$ $\left.\left.\sum_{k=4 n+4 p+7}(k+k+1)\right\}\right\}$

$=4 \sum_{m \geq 1}\left\{(2 m-1)(4 m+4 n+1)+(m+1) \sum_{k=1}^{\frac{n-m+3}{2}}(8 k+4 m+4 n-5)+(m+\right.$ 1) $\left.\sum_{k=1}^{\frac{n-m+1}{2}}\{(8 k+4 m+4 n-3)+2(8 k+4 m+4 n-1)+2(8 k+4 m+4 n+1)\}\right\}+$ $4 \sum_{m \geq 2} \sum_{p=0}^{m-2}\{(m-p)\{2(4 n+4 p+4)+1+2(2(4 n+4 p+5)+1)\}+(m-p-$ 1) $\{2(2(4 n+4 p+6)+1)+2(4 n+4 p+7)+1\}\}$

$=4 \sum_{m \geq 1}\left\{(2 m-1)(4 m+4 n+1)+(m+1) \sum_{k=1}^{\frac{n-m+3}{2}}(8 k+4 m+4 n-5)+(m+\right.$ 1) $\left.\sum_{k=1}^{\frac{n-m+1}{2}}\{8 k+4 m+4 n-3+16 k+8 m+8 n-2+16 k+8 m+8 n+2\}\right\}+$ $4 \sum_{m \geq 2} \sum_{p=0}^{m-2}\{(m-p)\{8 n+8 p+8+1+16 n+16 p+20+2\}+(m-p-1)\{16 n+$ $16 p+24+2+8 n+8 p+14+1\}\}$.

Finally, for all $m, n \in N$, where $m$ and $n$ have opposite pairity, the first Zagreb eccentricity index of bismuth tri-iodide $G(m, n)$ is

$$
\begin{aligned}
& M_{1}^{*}(G(m, n))=4 \sum_{m \geq 1}\left\{(2 m-1)(4 m+4 n+1)+(m+1) \sum_{k=1}^{\frac{n-m+3}{2}}(8 k+4 m+\right. \\
& \left.4 n-5)+(m+1) \sum_{k=1}^{\frac{n-m+1}{2}}(40 k+20 m+20 n-3)\right\}+4 \sum_{m \geq 2} \sum_{p=0}^{m-2} \\
& \{(m-p)(48 n+48 p+72)-(24 n+24 p+41)\} .
\end{aligned}
$$

Theorem 2. Let $G(m, n)$, for all $m, n \in N$, where $m$ and $n$ have same pairity, be the bismuth tri-iodide, then the first Zagreb eccentricity index $M_{1}^{*}$ of $G(m, n)$ is:

$$
\begin{aligned}
& M_{1}^{*}(G(m, n))=4 \sum_{m \geq 1}\left\{(2 m+1)(4 m+4 n+3)+(m+1) \sum_{k=1}^{\frac{n-m+2}{2}}(24 k\right. \\
& \left.+12 m+12 n-7)+(m+1) \sum_{k=1}^{\frac{n-m}{2}}(24 k+12 m+12 n+7)\right\}+4 \sum_{m \geq 2}\{(m- \\
& \text { 1) } \left.(4 m+4 n+1)+\sum_{p=0}^{m-2}\{(m-p)(48 n+48 p+72)-(24 n+24 p+41)\}\right\} .
\end{aligned}
$$

Proof. Let $G(m, n)$, where $m$ and $n$ have same pairity, be the bismuth tri-iodide contains $2(4 m n+5 n+5 m+1)$ vertices and $12(m+m n+n)$ edges.

The formula of first Zagreb eccentricity index is

$$
M_{1}^{*}(G)=\sum_{u v \in E(G)}[\varepsilon(u)+\varepsilon(v)] .
$$

Using the edge partitioned from Table 2, we have the following computations $M_{1}^{*}\left(G(m, n)=\sum_{m \geq 1}\left\{4(2 m+1) \sum_{k=2 m+2 n+1}(k+k+1)+4(m+1) \sum_{k=1}^{\frac{n-m+2}{2}}\{2(4 k\right.\right.$ $+2 m+2 n-2+4 k+2 m+2 n-1)+(4 k+2 m+2 n-1+4 k+2 m+2 n)\}+4(m+$ 1) $\sum_{k=1}^{\frac{n-m}{2}}\{(4 k+2 m+2 n+4 k+2 m+2 n+1)+2(4 k+2 m+2 n+1+4 k+2 m+2 n+$ 
$2)\}\}+4 \sum_{m \geq 2}\left\{(m-1) \sum_{k=2 m+2 n}(k+k+1)+\sum_{p=0}^{m-2}\left\{(m-p)\left\{\sum_{k=4 n+4 p+4}(k+\right.\right.\right.$ $\left.k+1)+2 \sum_{k=4 n+4 p+5}(k+k+1)\right\}+(m-p-1)\left\{2 \sum_{k=4 n+4 p+6}(k+k+1)+\right.$ $\left.\left.\sum_{k=4 n+4 p+7}(k+k+1)\right\}\right\}$

$=4 \sum_{m \geq 1}\left\{(2 m+1)(4 m+4 n+2+1)+(m+1) \sum_{k=1}^{\frac{n-m+2}{2}}\{2(8 k+4 m+4 n-3)+\right.$ $(8 k+4 m+4 n-1)\}+(m+1) \sum_{k=1}^{\frac{n-m}{2}}\{(8 k+4 m+4 n+1)+2(8 k+4 m+4 n+$ $3)\}\}+4 \sum_{m \geq 2}\left\{(m-1)(4 m+4 n+1)+\sum_{p=0}^{m-2}\{(m-p)\{2(4 n+4 p+4)+1+\right.$ $2(2(4 n+4 p+5)+1)\}+(m-p-1)\{2(2(4 n+4 p+6)+1)+2(4 n+4 p+7)+1\}\}$ $=4 \sum_{m \geq 1}\left\{(2 m+1)(4 m+4 n+3)+(m+1) \sum_{k=1}^{\frac{n-m+2}{2}}\{16 k+8 m+8 n-6+8 k+\right.$ $\left.4 m+4 n-1\}+(m+1) \sum_{k=1}^{\frac{n-m}{2}}\{8 k+4 m+4 n+1+16 k+8 m+8 n+6\}\right\}+$ $4 \sum_{m \geq 2}\left\{(m-1)(4 m+4 n+1)+\sum_{p=0}^{m-2}\{(m-p)\{8 n+8 p+8+1+16 n+16 p+\right.$ $20+2\}+(m-p-1)\{16 n+16 p+24+2+8 n+8 p+14+1\}\}$.

Finally, for all $m, n \in N$, where $m$ and $n$ have same pairity, the first Zagreb eccentricity index of bismuth tri-iodide $G(m, n)$ is $M_{1}^{*}(G(m, n))=4 \sum_{m \geq 1}\left\{(2 m+1)(4 m+4 n+3)+(m+1) \sum_{k=1}^{\frac{n-m+2}{2}}(24 k\right.$
$\left.+12 m+12 n-7)+(m+1) \sum_{k=1}^{\frac{n-m}{2}}(24 k+12 m+12 n+7)\right\}+4 \sum_{m \geq 2}\{(m-$
$\left.1)(4 m+4 n+1)+\sum_{p=0}^{m-2}\{(m-p)(48 n+48 p+72)-(24 n+24 p+41)\}\right\}$.

\subsection{Eccentricity based second Zagreb index}

In this section we find the second Zagreb eccentricity index of Bismuth tri-iodide $M_{1}^{* *}(G(m, n))$.

Theorem 3. Let $G(m, n)$, for all $m, n \in N$, where $m$ and $n$ have opposite pairity, be the bismuth tri-iodide, then the second Zagreb eccentricity index $M_{1}^{* *}$ of $G(m, n)$ is

$M_{1}^{* *}(G(m, n))=2 \sum_{m \geq 1}\left\{12 m(m+n)^{2}-4 n(m+n)+m+8(m+1) \sum_{k=1}^{\frac{n-m+3}{2}}(2 k+\right.$ $\left.m+n-1)^{2}+(m+1) \sum_{k=m+n+1}^{2 n+1}(2 k+1)^{2}+8(2 m+3) \sum_{k=1}^{\frac{n-m+1}{2}}(2 k+m+n)^{2}\right\}+$ $2 \sum_{m \geq 2} \sum_{p=0}^{m-2}\left\{(m-p)\left\{128(n+p)^{2}+416(n+p)+346\right\}-\left\{48(n+p)^{2}+184(n+\right.\right.$ $p)+177\}\}$.

Proof. Let $G(m, n)$, where $m$ and $n$ have opposite pairity, be the bismuth tri-iodide contains $2(4 m n+5 n+5 m+1)$ vertices and $12(m+m n+n)$ edges.

The general formula of second Zagreb eccentricity index is

$$
M_{1}^{* *}(G)=\sum_{v \in V(G)}[\varepsilon(v)]^{2} .
$$


Using the vertex partitioned from Table 3, we have the following computations $M_{1}^{* *}(G(m, n))=\sum_{m \geq 1}\left\{2(2 m-1) \sum_{k=2 m+2 n}(k)^{2}+2 m \sum_{k=2 m+2 n+1}\right.$ $(k)^{2}+4(m+1) \sum_{k=1}^{\frac{n-m+3}{2}}(4 k+2 m+2 n-2)^{2}+2(m+1) \sum_{k=m+n+1}^{2 n+1}(2 k+1)^{2}+$ $\left.4(2 m+3) \sum_{k=1}^{\frac{n-m+1}{2}}(4 k+2 m+2 n)^{2}\right\}+\sum_{m \geq 2}\left\{\sum_{p=0}^{m-2}(m-p)\left\{2 \sum_{k=4 n+4 p+5}(k)^{2}+\right.\right.$ $\left.8 \sum_{k=4 n+4 p+6}(k)^{2}\right\}+\sum_{p=0}^{m-2}(m-p-1)$ $\left.\left\{2 \sum_{k=4 n+4 p+7}(k)^{2}+4 \sum_{k=4 n+4 p+8}(k)^{2}\right\}\right\}$ $=2 \sum_{m \geq 1}\left\{(2 m-1)(2 m+2 n)^{2}+2 m(2 m+2 n+1)^{2}+8(m+1) \sum_{k=1}^{\frac{n-m+3}{2}}(2 k+\right.$ $\left.m+n-1)^{2}+(m+1) \sum_{k=m+n+1}^{2 n+1}(2 k+1)^{2}+8(2 m+3) \sum_{k=1}^{\frac{n-m+1}{2}}(2 k+m+n)^{2}\right\}+$ $2 \sum_{m \geq 2} \sum_{p=0}^{m-2}\left\{(m-p)\left\{(4 n+4 p+5)^{2}+4(4 n+4 p+6)^{2}\right\}+(m-p-1)\{(4 n+\right.$ $\left.\left.4 p+7)^{2}+2(4 n+4 p+8)^{2}\right\}\right\}$

$=2 \sum_{m \geq 1}\left\{12 m^{3}+24 m^{2} n+12 m n^{2}-4 m n-4 n^{2}+m+8(m+1) \sum_{k=1}^{\frac{n-m+3}{2}}(2 k+\right.$ $\left.m+n-1)^{2}+(m+1) \sum_{k=m+n+1}^{2 n+1}(2 k+1)^{2}+8(2 m+3) \sum_{k=1}^{\frac{n-m+1}{2}}(2 k+m+n)^{2}\right\}+$ $2 \sum_{m \geq 2} \sum_{p=0}^{m-2}\left\{(m-p)\left\{80 n^{2}+80 p^{2}+169+160 n p+232 p+232 n\right\}+(m-p-\right.$ 1) $\left.\left\{48 n^{2}+48 p^{2}+177+96 n p+184 p+184 n\right\}\right\}$.

Finally, for all $m, n \in N$, where $m$ and $n$ have opposite pairity, the second Zagreb eccentricity index of bismuth tri-iodide $G(m, n)$ is $M_{1}^{* *}(G(m, n))=2 \sum_{m \geq 1}\left\{12 m(m+n)^{2}-4 n(m+n)+m+8(m+1) \sum_{k=1}^{\frac{n-m+3}{2}}(2 k+\right.$ $\left.m+n-1)^{2}+(m+1) \sum_{k=m+n+1}^{2 n+1}(2 k+1)^{2}+8(2 m+3) \sum_{k=1}^{\frac{n-m+1}{2}}(2 k+m+n)^{2}\right\}+$ $2 \sum_{m \geq 2} \sum_{p=0}^{m-2}\left\{(m-p)\left\{128(n+p)^{2}+416(n+p)+346\right\}-\left\{48(n+p)^{2}+184(n+\right.\right.$ $p)+177\}\}$.

Theorem 4. Let $G(m, n)$, for all $m, n \in N$, where $m$ and $n$ have same pairity, be the bismuth tri-iodide, then the second Zagreb eccentricity index $M_{1}^{* *}$ of $G(m, n)$ is

$M_{1}^{* *}(G(m, n))=2 \sum_{m \geq 1}\left\{20 m(m+n)^{2}+4 m(13 m+17 n)+49 m+16(n+\right.$ $1)^{2}+(m+1) \sum_{k=m+n+1}^{2 n+1}(2 k+1)^{2}+8(m+1) \sum_{k=1}^{\frac{n-m+2}{2}}(2 k+m+n)^{2}+8(2 m+$ 3) $\left.\sum_{k=1}^{\frac{n-m}{2}}(2 k+m+n+1)^{2}\right\}+2 \sum_{m \geq 2}\left\{4(m-1)(m+n)^{2}+\sum_{p=0}^{m-2}\{(m-p)\{128(n+\right.$ $\left.\left.\left.p)^{2}+416(n+p)+346\right\}-\left\{48(n+p)^{2}+184(n+p)+177\right\}\right\}\right\}$.

Proof. Let $G(m, n)$, where $m$ and $n$ have same pairity, be the bismuth tri-iodide contains $2(4 m n+5 n+5 m+1)$ vertices and $12(m+m n+n)$ edges. 
The general formula of second Zagreb eccentricity index is

$$
M_{1}^{* *}(G)=\sum_{v \in V(G)}[\varepsilon(v)]^{2} .
$$

Using the vertex partitioned from Table 4, we have the following computations $M_{1}^{* *}(G(m, n))=\sum_{m \geq 1}\left\{2 m \sum_{k=2 m+2 n+1}(k)^{2}+8(m+1) \sum_{k=2 m+2 n+2}\right.$

$(k)^{2}+2(m+1) \sum_{k=m+n+1}^{2 n+1}(2 k+1)^{2}+4(m+1) \sum_{k=1}^{\frac{n-m+2}{2}}(4 k+2 m+2 n)^{2}+4(2 m+$ 3) $\left.\sum_{k=1}^{\frac{n-m}{2}}(4 k+2 m+2 n+2)^{2}\right\}+\sum_{m \geq 2}\left\{2(m-1) \sum_{k=2 m+2 n}\right.$

$(k)^{2}+\left\{\sum_{p=0}^{m-2}(m-p)\left\{2 \sum_{k=4 n+4 p+5}(k)^{2}+8 \sum_{k=4 n+4 p+6}(k)^{2}\right\}+\sum_{p=0}^{m-2}\right.$

$\left.\left.(m-p-1)\left\{2 \sum_{k=4 n+4 p+7}(k)^{2}+4 \sum_{k=4 n+4 p+8}(k)^{2}\right\}\right\}\right\}$

$=2 \sum_{m \geq 1}\left\{m(2 m+2 n+1)^{2}+4(m+1)(2 m+2 n+2)^{2}+(m+1) \sum_{k=m+n+1}^{2 n+1}(2 k+\right.$ $\left.1)^{2}+8(m+1) \sum_{k=1}^{\frac{n-m+2}{2}}(2 k+m+n)^{2}+8(2 m+3) \sum_{k=1}^{\frac{n-m}{2}}(2 k+m+n+1)^{2}\right\}+$ $2 \sum_{m \geq 2}\left\{(m-1)(2 m+2 n)^{2}+\sum_{p=0}^{m-2}\left\{(m-p)\left\{(4 n+4 p+5)^{2}+4(4 n+4 p+6)^{2}\right\}+\right.\right.$ $\left.(m-p-1)\left\{(4 n+4 p+7)^{2}+2(4 n+4 p+8)^{2}\right\}\right\}$

$=2 \sum_{m \geq 1}\left\{20 m^{3}+40 m^{2} n+20 m n^{2}+68 m n+52 m^{2}+68 m n+49 m+32 n+\right.$ $16 n^{2}+16+(m+1) \sum_{k=m+n+1}^{2 n+1}(2 k+1)^{2}+8(m+1) \sum_{k=1}^{\frac{n-m+2}{2}}(2 k+m+n)^{2}+$ $\left.8(2 m+3) \sum_{k=1}^{\frac{n-m}{2}}(2 k+m+n+1)^{2}\right\}+2 \sum_{m \geq 2}\left\{4(m-1)(m+n)^{2}+\sum_{p=0}^{m-2}\{(m-\right.$ p) $\left\{80 n^{2}+80 p^{2}+169+160 n p+232 p+232 n\right\}+(m-p-1)\left\{48 n^{2}+48 p^{2}+177+\right.$ $96 n p+184 p+184 n\}\}$.

Finally, for all $m, n \in N$, where $m$ and $n$ have same pairity, the second Zagreb eccentricity index of bismuth tri-iodide $G(m, n)$ is

$M_{1}^{* *}(G(m, n))=2 \sum_{m \geq 1}\left\{20 m(m+n)^{2}+4 m(13 m+17 n)+49 m+16(n+\right.$ $1)^{2}+(m+1) \sum_{k=m+n+1}^{2 n+1}(2 k+1)^{2}+8(m+1) \sum_{k=1}^{\frac{n-m+2}{2}}(2 k+m+n)^{2}+8(2 m+$ 3) $\left.\sum_{k=1}^{\frac{n-m}{2}}(2 k+m+n+1)^{2}\right\}+2 \sum_{m \geq 2}\left\{4(m-1)(m+n)^{2}+\sum_{p=0}^{m-2}\{(m-p)\{128(n+\right.$ $\left.\left.\left.p)^{2}+416(n+p)+346\right\}-\left\{48(n+p)^{2}+184(n+p)+177\right\}\right\}\right\}$.

\subsection{Eccentricity based third Zagreb index}

In this section we find the third Zagreb eccentricity index of Bismuth tri-iodide $M_{2}^{*}(G(m, n))$.

Theorem 5. Let $G(m, n)$, for all $m, n \in N$, where $m$ and $n$ have opposite pairity, be the bismuth tri-iodide, then the third Zagreb eccentricity index $M_{2}^{*}$ of $G(m, n)$ is

$M_{2}^{*}(G(m, n))=8 \sum_{m \geq 1}\left\{(2 m-1)(m+n)(2 m+2 n+1)+(m+1) \sum_{k=1}^{\frac{n-m+3}{2}}\{2(2 k+\right.$ 


$$
\begin{aligned}
& \left.m+n)^{2}-5(2 k+m+n)+3\right\}+(m+1) \sum_{k=1}^{\frac{n-m+1}{2}}\{10 \\
& \left.\left.(2 k+m+n)^{2}-3(2 k+m+n)+1\right\}\right\}+16 \sum_{m \geq 2} \sum_{p=0}^{m-2}\left\{( m - p ) \left\{24(n+p)^{2}+\right.\right. \\
& \left.72(n+p)+55\}-\left\{12(n+p)^{2}+41(n+p)+35\right\}\right\} .
\end{aligned}
$$

Proof. Let $G(m, n)$, where $m$ and $n$ have opposite pairity, be the bismuth tri-iodide contains $2(4 m n+5 n+5 m+1)$ vertices and $12(m+m n+n)$ edges.

The general formula of third Zagreb eccentricity index is

$$
M_{2}^{*}(G)=\sum_{u v \in E(G)}[\varepsilon(u) \cdot \varepsilon(v)] .
$$

Using the edge partitioned from Table 1, we have the following computations $M_{2}^{*}\left(G(m, n)=\sum_{m \geq 1}\left\{4(2 m-1) \sum_{k=2 m+2 n}(k)(k+1)+4(m+1)\right.\right.$ $\sum_{k=1}^{\frac{n-m+3}{2}}(4 k+2 m+2 n-3)(4 k+2 m+2 n-2)+4(m+1) \sum_{k=1}^{\frac{n-m+1}{2}}\{(4 k+$ $2 m+2 n-2)(4 k+2 m+2 n-1)+2(4 k+2 m+2 n-1)(4 k+2 m+2 n)+2(4 k+$ $2 m+2 n+1)(4 k+2 m+2 n)\}\}+4 \sum_{m \geq 2} \sum_{p=0}^{m-2}\left\{(m-p)\left\{\sum_{k=4 n+4 p+4}(k)(k+1)+\right.\right.$ $\left.2 \sum_{k=4 n+4 p+5}(k)(k+1)\right\}+(m-p-1)\left\{2 \sum_{k=4 n+4 p+6}(k)(k+1)+\sum_{k=4 n+4 p+7}(k)(k+\right.$ 1) $\}\}$

$=4 \sum_{m \geq 1}\left\{(2 m-1)(2 m+2 n)(2 m+2 n+1)+2(m+1) \sum_{k=1}^{\frac{n-m+3}{2}}(4 k+2 m+\right.$ $2 n-3)(2 k+m+n-1)+2(m+1) \sum_{k=1}^{\frac{n-m+1}{2}}\{(4 k+2 m+2 n-1)(6 k+3 m+$ $3 n-1)+(4 k+2 m+2 n)(4 k+2 m+2 n+1)\}\}+4 \sum_{m \geq 2} \sum_{p=0}^{m-2}\{(m-p)\{(4 n+$ $4 p+4)(4 n+4 p+5)+2(4 n+4 p+5)(4 n+4 p+6)\}+(m-p-1)\{2(4 n+4 p+$ $6)(4 n+4 p+7)+(4 n+4 p+7)(4 n+4 p+8)\}\}$ $=8 \sum_{m \geq 1}\left\{(2 m-1)(m+n)(2 m+2 n+1)+(m+1) \sum_{k=1}^{\frac{n-m+3}{2}}\left\{2(2 k+m+n)^{2}-\right.\right.$ $5(2 k+m+n)+3\}+(m+1) \sum_{k=1}^{\frac{n-m+1}{2}}\left\{40 k^{2}+10 m^{2}+10 n^{2}-6 k+40 k m+20 m n+\right.$ $40 k n-3 m-3 n+1\}\}+16 \sum_{m \geq 2} \sum_{p=0}^{m-2}\left\{(m-p)\left\{12 n^{2}+12 p^{2}+24 n p+31 n+\right.\right.$ $\left.31 p+20\}+(m-p-1)\left\{12 n^{2}+12 p^{2}+24 n p+41 n+41 p+35\right\}\right\}$.

Finally, for all $m, n \in N$, where $m$ and $n$ have opposite pairity, the third Zagreb eccentricity index of bismuth tri-iodide $G(m, n)$ is $M_{2}^{*}(G(m, n))=8 \sum_{m \geq 1}\left\{(2 m-1)(m+n)(2 m+2 n+1)+(m+1) \sum_{k=1}^{\frac{n-m+3}{2}}\{2(2 k+\right.$
$\left.m+n)^{2}-5(2 k+m+n)+3\right\}+(m+1) \sum_{k=1}^{\frac{n-m+1}{2}}\{10$
$\left.\left.(2 k+m+n)^{2}-3(2 k+m+n)+1\right\}\right\}+16 \sum_{m \geq 2} \sum_{p=0}^{m-2}\left\{(m-p)\left\{24(n+p)^{2}+\right.\right.$
$\left.72(n+p)+55\}-\left\{12(n+p)^{2}+41(n+p)+35\right\}\right\}$.

Theorem 6. Let $G(m, n)$, for all $m, n \in N$, where $m$ and $n$ have same pairity, be the bismuth tri-iodide, then the third Zagreb eccentricity index $M_{2}^{*}$ 
of $G(m, n)$ is

$M_{2}^{*}(G(m, n))=8 \sum_{m \geq 1}\left\{(2 m+1)(m+n+1)(2 m+2 n+1)+(m+1) \sum_{k=1}^{\frac{n-m+2}{2}}\{6(2 k+\right.$ $\left.m+n)^{2}-7(2 k+m+n)+2\right\}+(m+1) \sum_{k=1}^{\frac{n-m}{2}}\left\{6(2 k+m+n)^{2}+7(2 k+m+\right.$ $n)+2\}\}+8 \sum_{m \geq 2}\left\{(m-1)(m+n)(2 m+2 n+1)+2 \sum_{p=0}^{m-2}\{(m-p)\{24(n+\right.$ $\left.\left.\left.p)^{2}+72(n+p)+55\right\}-\left\{12(n+p)^{2}+41(n+p)+35\right\}\right\}\right\}$.

Proof. Let $G(m, n)$, where $m$ and $n$ have same pairity, be the bismuth tri-iodide contains $2(4 m n+5 n+5 m+1)$ vertices and $12(m+m n+n)$ edges.

The general formula of third Zagreb eccentricity index is

$$
M_{2}^{*}(G)=\sum_{u v \in E(G)}[\varepsilon(u) \cdot \varepsilon(v)] .
$$

Using the edge partitioned from Table 2, we have the following computations: $M_{2}^{*}\left(G(m, n)=\sum_{m>1}\left\{4(2 m+1) \sum_{k=2 m+2 n+1}(k)(k+1)+4(m+1) \sum_{k=1}^{\frac{n-m+2}{2}}\{2(4 k+\right.\right.$ $2 m+2 n-2)(4 k+2 m+2 n-1)+(4 k+2 m+2 n-1)(4 k+2 m+2 n)\}+4(m+$ 1) $\sum_{k=1}^{\frac{n-m}{2}}\{(4 k+2 m+2 n)(4 k+2 m+2 n+1)+2(4 k+2 m+2 n+1)(4 k+2 m+2 n+$ $2)\}\}+4 \sum_{m \geq 2}\left\{(m-1) \sum_{k=2 m+2 n}(k)(k+1)+\sum_{p=0}^{m-2}\left\{(m-p)\left\{\sum_{k=4 n+4 p+4}(k)(k+\right.\right.\right.$ $\left.1)+2 \sum_{k=4 n+4 p+5}(k)(k+1)\right\}+(m-p-1)\left\{2 \sum_{k=4 n+4 p+6}(k)(k+1)\right.$

$\left.\left.\left.+\sum_{k=4 n+4 p+7}(k)(k+1)\right\}\right\}\right\}$

$=4 \sum_{m \geq 1}\left\{(2 m+1)(2 m+2 n+1)(2 m+2 n+2)+2(m+1) \sum_{k=1}^{\frac{n-m+2}{2}}\{(4 k\right.$

$+2 m+2 n-1)(4 k+2 m+2 n-2+2 k+m+n)\}+2(m+1) \sum_{k=1}^{\frac{n-m}{2}}\{(4 k+$ $2 m+2 n+1)(6 k+3 m+3 n+2)\}\}+4 \sum_{m>2}\{(m-1)(2 m+2 n)(2 m+2 n+1)+$ $\sum_{p=0}^{m-2}\{(m-p)\{(4 n+4 p+4)(4 n+4 p+5)+2(4 n+4 p+5)(4 n+4 p+6)\}+$ $(m-p-1)\{2(4 n+4 p+6)(4 n+4 p+7)+(4 n+4 p+7)(4 n+4 p+8)\}\}\}$ $=8 \sum_{m \geq 1}\left\{(2 m+1)(m+n+1)(2 m+2 n+1)+(m+1) \sum_{k=1}^{\frac{n-m+2}{2}}\left\{24 k^{2}+6 m^{2}+6 n^{2}+\right.\right.$ $24 k m+12 m n+24 k n-14 k-7 m-7 n+2\}+(m+1) \sum_{k=1}^{\frac{n-m}{2}}\left\{24 k^{2}+6 m^{2}+6 n^{2}+\right.$ $24 k m+12 m n+24 k n+14 k+7 m+7 n+2\}\}+16 \sum_{m \geq 2} \sum_{p=0}^{m-2}\left\{(m-p)\left\{12 n^{2}+\right.\right.$ $\left.\left.\left.12 p^{2}+24 n p+31 n+31 p+20\right\}+(m-p-1)\left\{12 n^{2}+12 p^{2}+24 n p+41 n+41 p+35\right\}\right\}\right\}$. Finally, for all $m, n \in N$, where $m$ and $n$ have same pairity, the third Zagreb eccentricity index of bismuth tri-iodide $G(m, n)$ is

$M_{2}^{*}(G(m, n))=8 \sum_{m \geq 1}\left\{(2 m+1)(m+n+1)(2 m+2 n+1)+(m+1) \sum_{k=1}^{\frac{n-m+2}{2}}\{6(2 k\right.$ $\left.+m+n)^{2}-7(2 k+m+n)+2\right\}+(m+1) \sum_{k=1}^{\frac{n-m}{2}}\left\{6(2 k+m+n)^{2}+7(2 k+m+\right.$ $n)+2\}\}+8 \sum_{m \geq 2}\left\{(m-1)(m+n)(2 m+2 n+1)+2 \sum_{p=0}^{m-2}\{(m-p)\{24(n+\right.$ $\left.\left.\left.p)^{2}+72(n+p)+55\right\}-\left\{12(n+p)^{2}+41(n+p)+35\right\}\right\}\right\}$. 
Acknowledgement. This work was supported by the Natural Science Foundation of the Education Department of Anhui Province (KJ2018A0598).

\section{References}

[1] A.Q. Baig, M. Imran, H. Ali, On topological indices of poly oxide, poly silicate, DOX, and DSL networks, Canadian J. of Chemistry, 93 (2015), $730-739$.

[2] B. Rajan, A. William, C. Grigorious, S. Stephen, On certain topological indices of silicate, Honeycomb and Hexagonal networks, J. Comp. and Math. Sci., 3, No 5 (2012), 530-535.

[3] B. Zhou, I. Gutman, Further properties Of Zagreb indices, MATCH Commun. Math. Comput. Chem., 54 (2005), 233-239.

[4] D. Vukiçević, A. Graovac, Note on the comparison of the first and second normalized Zagreb eccentricity indices, Acta Chim. Slov., 57 (2010), 524528.

[5] D. Nason, L. Keller, The growth and crystallography of bismuth tri-iodide crystals grown by vapor transport, J. of Crystal Growth (1995), 156-221.

[6] H. Yorikawa, S. Muramatsu, Theoretical study of crystal and electronic structures of $\mathrm{BiI}_{3}$, J. of Physics: Condensed Matter, 20 (2008), ID 325220.

[7] K.C. Das, On comparing Zagreb indices of graphs, MATCH Commun. Math. Comput. Chem., 63 (2010), 433-440.

[8] K.C. Das, Some properties of the Zagreb eccentricity indices, Ars Math. Cont., 6 (2013), 117-125.

[9] K.C. Das, I. Gutman, Some properties of the second Zagreb index, MATCH Commun. Math. Comput. Chem., 52 (2004), 103-112.

[10] K. Watanabe, T. Karasawa, T. Komatsu, Y. Kaifu, Optical properties of extrinsic two-dimensional excitons in $\mathrm{BiI}_{3}$ single crystals, J. of Phys. Soc. of Japan, 55 (1986), 897.

[11] M. Ghorbani, M.A. Hosseinzadeh, A new version of Zagreb indices, Filomat, 26), No 1 (2012), 93-100. 
[12] M. Imran, S. Hayat, On computation of topological indices of aztec diamonds, Sci. Int. (Lahore), 26, No 4 (2014), 1407-1412.

[13] M. Schluter, M.L. Cohen, S.E. Kohn, C.Y. Fong, Electronic structure of BiI $I_{3}$, Physica Status Solidi (B), 78 (1976), 737.

[14] M.R. Farahani, Computing fourth atom bond connectivity index of Vphenylenic nanotubes and nanotori, Acta Chimica Slovenica, 60, No 2 (2013), 429-432.

[15] R.W.G. Wyckoff, Crystal Structures, John Wiley and Sons, Inc., New York, London, Sydney (1964).

[16] S. Hayat, M. Imran, Computation of topological indices of certain network, Appl. Math. and Computation, 240 (2014), 213-228.

[17] S. Hayat, M.A. Malik, M. Imran, Computing topological indices of honeycomb derived networks, Romanian J. of Information Sci. and Techn., 18 (2015), 144-165.

[18] S. Hayat, M. Imran, Computation of certain topological indices of nanotubes covered by $C_{5}$ and $C_{7}, J$. Comput. Theor. Nanosci., 12, No 4 (2015), $533-541$.

[19] W. Gao, W. Wang, M.K. Jamil, M.R. Farahani, Electron energy studying of molecular structures via forgotten topological index computation, J. of Chemistry, (2016), Article ID 1053183, 7 pp., doi: 10.1155/2016/1053183.

[20] W. Gao, W.F. Wang, M.K. Jamil, R. Farooq, M.R. Farahani, Generalized atom-bond connectivity analysis of several chemical molecular graphs, Bulgarian Chemical Communications, 48, No 3 (2016), 543-549. 